\title{
Aspectos Bionômicos de Squamatoides trivittatus (Diptera, Sarcophagidae) sob Condições de Laboratório
}

\author{
Ricardo JB Salviano, Rubens Pinto de Mello*, Lilian CNH Beck, \\ José Mario d'Almeida
}

\author{
Laboratório de Biologia e Controle de Insetos Vetores, Departamento de Biologia *Laboratório de Diptera, \\ Departamento de Entomologia, Instituto Oswaldo Cruz, Av. Brasil 4365, 21045-900 \\ Rio de Janeiro, RJ, Brasil
}

\begin{abstract}
Bionomical aspects of de Squamatoides trivittatus (Diptera, Sarcophagidae) under laboratory conditions - Experiments with Squamatoides trivitattus were carried out in two different controlled temperatures $\left(16 \pm 1{ }^{\circ} \mathrm{C} / 50-60 \% \mathrm{RH}\right.$ and $\left.27 \pm 1{ }^{\circ} \mathrm{C} / 70-80 \% \mathrm{RH}\right)$. The viability of larvae and pupae at $27^{\circ} \mathrm{C}$ was $89.82 \%$ and $92.75 \%$ respectively. Larvae did not develop at $16^{\circ} \mathrm{C}$. Larval development lasted for $20 \pm 4 \mathrm{hr}, 16 \pm 8 \mathrm{hr}$ and $60 \pm 7 \mathrm{hr}$ for the first, second and third instars, respectively, completing a total of $96 \pm 6 \mathrm{hr}$. The mean pupal period lasted for $15.7 \pm 1.6$ days. In longevity tables for the adults, life-expectancy for $50 \%$ of the colony submitted to $16^{\circ} \mathrm{C}$ was of 1.78 weeks for males and 2.42 for females. At $27^{\circ} \mathrm{C}$ a life-expectancy of 1.15 weeks for males and 0.78 week for females was recorded. The average life-spans for males and females at $16^{\circ} \mathrm{C}$ were $3.5 \pm 2.0$ and $3.8 \pm 2.6$ weeks, respectively, and $1.9 \pm 1.2$ weeks for both sexes. At $27^{\circ} \mathrm{C}$, the longevity recorded was of $2.1 \pm 1.3$ weeks for males and $1.7 \pm 1.1$ week for females.
\end{abstract}

Key words: Diptera - Sarcophagidae - Squamatoides trivittatus - life history - Brazil

O gênero Squamatoides Curran, 1927 está representado por três espécies: $S$. trivittatus Curran, 1927, S. spitzi (Lopes 1933) e S. stahli Dodge, 1966, sendo que apenas as duas primeiras são encontradas no Brasil. S. trivittatus está restrita ao Campo Cerrado da América do Sul; no Brasil é encontrada nos estados de Mato Grosso, Goiás, Minas Gerais e São Paulo (Lopes 1969).

Linhares (1981), estudando dípteros muscóides, em São Paulo, verificou que $S$. trivittatus apresenta um elevado grau de sinantropia. Em áreas que apresentam condições ambientais muito próximas à estudada por Linhares, Mendes (1991) obteve 33,33\% dos espécimens coletados em fezes humanas, o que confere importância a este Sarcophagidae como potencial vetor de agentes enteropatógenos.

Apesar do interesse médico e sanitário dos dípteros desta família, são escassos os estudos sobre sua biologia. Em sua maioria, os trabalhos relacionados com as fases imaturas avaliam o período de desenvolvimento, a viabilidade e a competição larval em diversas condições de temperatura e

Recebido em 21 de julho de 1995 Aceito em 22 de novembro de 1995 umidade relativa (Kamal 1958, House 1977, Levot et al. 1979, Baxter \& Morrison 1983; Jirón \& Bolanos 1986, Ferraz 1992a,b). Os artigos relacionados à fase adulta estão em grande parte relacionados aos aspectos comportamentais (preferência de substrato para postura e alimentação), biogeográficos (sinantropia), longevidade e capacidade reprodutiva (Kamal 1958, Ferreira 1978, Linhares 1979, 1981, d'Almeida 1983, 1984, 1986, 1988, 1989, 1993, Ferraz 1992a,b). Tais aspectos, dentre outros, podem ser analisados através de tabelas de vida, que nos permitem avaliar satisfatoriamente todo o processo de vida dos insetos e relacioná-lo a diversos fatores, como temperatura, dieta, efeito de grupo (Pearl \& Parker 1921, Pearl et al. 1927, Jackson 1936, Mendes 1961, Carey 1987, Bressan \& Teles 1991).

Devido à inexistência de trabalhos sobre a biologia de S. trivittatus, foram avaliados alguns aspectos de suas fases evolutivas: período e viabilidade larval e pupal, razão sexual, longevidade, curva de sobrevivência de adultos em diferentes temperaturas e umidades. Foram elaboradas tabelas de expectativa de vida.

\section{MATERIAIS E MÉTODOS}

Os experimentos foram realizados em câmaras climatizadas reguladas a $16 \pm 1^{\circ} \mathrm{C}$ e $27 \pm 1^{\circ} \mathrm{C}$ de temperatura e $50-60 \%$ e $70-80 \%$ de umidade relativa 
do ar (URA). Essas temperaturas foram escolhidas por corresponderem às médias das máximas e mínimas durante 27 anos, no Distrito Federal.

A colônia foi estabelecida a partir de insetos coletados em áreas de cerrado e urbana da cidade satélite Taguatinga (DF) no período de 27 de março a 04 de abril de 1992.

Para observação da viabilidade larval, utilizaram-se larvas de colônias mantidas em temperatura ambiente $\left(25-27^{\circ} \mathrm{C}\right)$. Setenta e oito grupos de 10 larvas de primeiro estádio foram introduzidos em recipientes plásticos $(5 \mathrm{~cm}$ altura x $6 \mathrm{~cm}$ diametro), contendo, cada um, $20 \mathrm{~g}$ de sardinha com $24 \mathrm{~h}$ de decomposição (2g/larva). Desses grupos, 20 foram levados para câmara climatizada a $16^{\circ} \mathrm{C}$ e os demais mantidos em câmara a $27^{\circ} \mathrm{C}$. As observações diárias foram feitas em dois horários: $8 \mathrm{~h}$ e $17 \mathrm{~h} 30 \mathrm{~min}$.

Para avaliar a duração do período larval foram utilizadas 720 larvas obtidas de larviposturas de uma colônia de indivíduos da sexta geração. Foram separados grupos de 20 larvas em 36 amostras. A cada duas horas uma amostra era retirada da câmara climatizada $\left(27^{\circ} \mathrm{C}\right.$ e $\left.70-80 \%\right)$ e as larvas eram sacrificadas e fixadas em San Jean (600 $\mathrm{ml}$ de água destilada, $300 \mathrm{ml}$ de álcool a 95\%, 100 $\mathrm{ml}$ de formalina e $10 \mathrm{ml}$ de ácido acético) a quente, e conservadas em álcool a 70\%, para posterior verificação do respectivo estádio.

Quanto à fase pupal (duração e viabilidade) e razão sexual, foram usadas 1264 larvas maduras recém-saídas dos substratos de criação. Após pesadas e individualizadas, 200 foram transferidas para a câmara climatizada a $16^{\circ} \mathrm{C}$ e as demais para câmara a $27^{\circ} \mathrm{C}$, registrando-se diariamente as que puparam, as pupas mortas e a emergência dos imagos.

Após emergência e sexagem, 14 amostras contendo 15 casais cada uma, foram separadas em dois grupos: o primeiro, contendo nove amostras, submetido a $27^{\circ} \mathrm{C}$ e o segundo, com cinco amostras, a $16^{\circ} \mathrm{C}$.

Como fonte diária de proteína e substrato de larviposição, utilizou-se sardinha, retirada do congelador, e mantida por $24 \mathrm{~h}$ em temperatura ambiente, fornecida, para cada gaiola, na proporção de $10 \mathrm{~g}$ por pote; além de água ad libitum e açúcar mascavo.

Registros diários do número de mortos em cada amostra foram efetuados com o objetivo de se construir as tabelas de vida, as curvas de sobrevivência e a estimativa das longevidades médias.

As longevidades para os dois tratamentos foram transformadas em $\sqrt{\mathrm{x}}$ e submetidas à análise das variâncias. As médias foram comparadas pelo teste "t" ao nível de significância de 5\%.

As tabelas de vida foram baseadas em Carey (1987) e Silveira Neto et al. (1976), que sugerem iniciar as mesmas com a projeção para 1000 exemplares, que é sua raiz. Os parâmetros estão organizados em colunas, em função do intervalo de idade (x), em semanas. Lx representa o número de sobreviventes no começo da idade $\mathrm{x}$; $\mathrm{dx}$ é a fração de insetos mortos entre as idades $\mathrm{x}$ e $\mathrm{x}+1$, estimada por $L_{x}-L_{x+1}$. Ex representa a estrutura etária, calculado por $\mathrm{L}_{x}+\mathrm{L}_{\mathrm{x}+1} / 2$. O valor de Tx obtemse pelo somatório de $E x+E_{x+1}+E_{x+2}+\ldots+E_{w}$, onde w é o último estágio. A esperança de vida (ex) foi estimada por $\mathrm{T}_{\mathrm{x}} / \mathrm{L}_{\mathrm{x}}$. 1000qx é a coluna de risco, estimada por $1000 \mathrm{~d}_{\mathrm{x}} / \mathrm{L}_{\mathrm{x}}$.

\section{RESULTADOS E DISCUSSÃO}

As larvas submetidas a $16^{\circ} \mathrm{C}$ só se desenvolveram até o segundo estádio. Tal fato pode ter ocorrido devido à exposição prolongada à temperatura e umidade baixas, provocando um ressecamento do substrato e, conseqüentemente, a morte das larvas; o mesmo foi observado durante a avaliação do período pupal, quando as 200 larvas maduras chegaram a pupar, mas não ocorreu emergência. Após três meses de observação, admitiu-se a hipótese de as pupas terem entrado em diapausa. Para esta avaliação, as pupas foram divididas em três grupos. No primeiro, através de dissecação avaliou-se o estado dos imagos, verificando a sua total desidratação. $\mathrm{O}$ segundo foi exposto à temperatura de $25^{\circ} \mathrm{C}$ e $60-70 \%$ de URA e o terceiro a $27^{\circ} \mathrm{C}$ e $80-90 \%$ de URA, e em ambos verificou-se que as larvas maduras chegaram a pupar, porém não emergiram devido à total desidratação do imago.

Em algumas espécies, a temperatura baixa não provoca a completa inviabilidade das fases imaturas, como em Parasarcophaga crassipalpis e Boettcherisca peregrina, que apresentam um período pupal mínimo de 56 e 50 dias, respectivamente, quando submetidos à temperatura de $15^{\circ} \mathrm{C}$ (Nishida 1984).

A $27^{\circ} \mathrm{C}$ a viabilidade média larval foi de $89,82 \%$, valor próximo aos obtidos por Madubunyl (1986) para Sarcophaga haemorrhoidalis $(80,69 \%$, a $23-28^{\circ} \mathrm{C}$ ). Resultados semelhantes também foram verificados para Peckia chrysostoma e Adiscochaeta ingens, submetidos à temperatura de $25,9^{\circ} \mathrm{C}$ e $79 \%$ de URA, com $97 \%$ e $93 \%$ de sobrevida, respectivamente (Ferraz 1992a).

O período larval para $S$. trivittatus a $27^{\circ} \mathrm{C} \mathrm{du}-$ rou $96 \pm 6 \mathrm{~h}$, onde o primeiro estádio teve duração de $20 \pm 4 \mathrm{~h}$, o segundo, de $16 \pm 8 \mathrm{~h}$ e o terceiro de $60 \pm 7$ h. O período larval do Sarcophagidae, $P$. 
chrysostoma, analisado por Ferraz (1992a), foi de $89 \mathrm{~h}$ a $25,9^{\circ} \mathrm{C}$ e $95 \mathrm{~h} \mathrm{a} 27^{\circ} \mathrm{C}$, bem próximo ao obtido para $S$. trivittatus.

Levot (1979) verificou que, a $27-28^{\circ} \mathrm{C}$, larvas de $P$. crassipalpis alcançaram o final do terceiro estádio após $69 \mathrm{~h}$, com peso máximo e mínimo de $235,7 \mathrm{mg}$ e $173,8 \mathrm{mg}$, respectivamente. Esta mesma espécie foi estudada por Nishida (1984), onde o período e peso máximo obtido em $25^{\circ} \mathrm{C}$ foi de oito dias e $152,6 \mathrm{mg}$; a $30^{\circ} \mathrm{C}$ chegou a sete dias com 135,9 mg. Para B. peregrina, esse autor obteve os seguintes pesos máximos e durações larvárias nas temperaturas de $25^{\circ} \mathrm{C}$ e $30^{\circ} \mathrm{C}$ : seis dias com 115,8 mg, e cinco dias com $87 \mathrm{mg}$, respectivamente.

A viabilidade pupal para $S$. trivittatus foi de 92,75\%. Kamal (1958) obteve resultados semelhantes para Sarcophaga spp. Madubunyl (1986), estudando Bercaea haemorrhoidalis, observou que a viabilidade pupal foi de $89,83 \%$ a $23-28^{\circ} \mathrm{C}$. De acordo com Ferraz (1992a) a $25,9^{\circ} \mathrm{C}$ e $79 \%$ de URA a viabilidade pupal para $P$. chrysostoma foi de $69,9 \%$ e para A. ingens $92,8 \%$.

O período pupal médio foi de $15,7 \pm 1,6$ dias, com o pico de emergência no 150 dia, para $37 \%$ das 1064 pupas. Dos adultos emergidos, 54,36\% foram machos e 45,64\% fêmeas; a emergência de adultos foi maior nas pupas que pesavam entre 200 e $299 \mathrm{mg}$ (Fig. 1). Os pesos máximos e mínimos das larvas que originaram fêmeas e machos foram de 320-120 mg e 370-150 mg, respectivamente. O peso médio para os machos foi de $257 \pm 33 \mathrm{mg}$ e $238 \pm 34 \mathrm{mg}$, para as fêmeas. A razão sexual foi de 0,6 nos imagos provenientes de larvas de 100-199 $\mathrm{mg}, 1,2$ para as larvas de $200-299 \mathrm{mg}$ e 1,8 para as larvas de 300-399 mg.

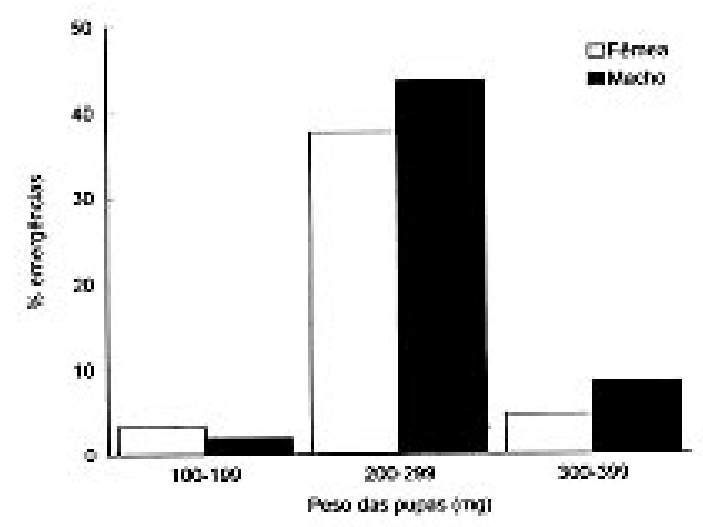

Fig. 1: relação entre a taxa de emergência de Squamatoides trivittatus e o peso das larvas maduras sob condições de temperatura e umidade constante $\left(27 \pm 1^{\circ} \mathrm{C}\right.$ e $70-80 \%$ de umidade relativa do ar).
Segundo Ferraz (1992a), o período pupal de $P$. chrysostoma e A. igens a $18^{\circ} \mathrm{C}$ foi $23,5 \pm 1,5$ dias e $33 \pm 3$ dias, respectivamente. O período pupal dos Sarcophagidae, em temperaturas próximas a $27^{\circ} \mathrm{C}$ geralmente fica na faixa de 10-20 dias (Nishida 1984, Jirón \& Bolanos 1986, Ferraz 1992a), o que concorda com os presentes resultados.

$\mathrm{Na}$ Tabela I são apresentadas as expectativas de vida para machos e fêmeas de $S$. trivittatus, nas temperaturas e umidades relativas propostas.

A expectativa de vida para 50\% da colônia submetida a $16 \pm 1^{\circ} \mathrm{C}$ e $50-60 \%$ de URA foi de 1,78 semanas para os machos e 2,42 semanas para as fêmeas. Já a colônia mantida a $27 \pm 1^{\circ} \mathrm{C}$ e $70-80 \%$ de URA foi de 1,15 semana para os machos e 0,78 semana para as fêmeas. Estes valores juntamente com os apresentados na Tabela II, deixam claro que à baixa temperatura, a fêmea de $S$. trivittatus apresenta uma longevidade maior do que as do macho, provavelmente devido a sua baixa atividade metabólica. A $27^{\circ} \mathrm{C}$ ocorreu o inverso onde as fêmeas apresentaram uma longevidade menor do que os machos, devido ao maior desgaste (aumento do metabolismo). Um dos aspectos que podem ter evidenciado este fato é o desgaste devido ao desenvolvimento ovariano, como também, podese sugerir que esta constatação esteja ligada a algumas características peculiares do ecossistema onde S. trivitattus ocorre. Estes resultados não corroboram com Mackerras (1933) que estudou fêmeas de várias espécies de moscas que vivem mais do que os machos. Ferraz (1992a) também obteve maior longevidade para fêmeas de $P$. chrysostoma e A. ingens .

A Fig. 2 apresenta as curvas de sobrevivência que tendem para o tipo III (Silveira Neto 1976, Carey 1987), onde dx decresce gradativamente a cada intervalo x (Tabela I). Analisando as curvas, pode-se verificar que a sobrevivência foi maior a $16^{\circ} \mathrm{C}$, onde $\mathrm{dx}$ aumentou rapidamente até a $4^{\mathrm{a}}$ semana, decaindo mais lentamente até a $7^{\mathrm{a}}$, quando aumentou novamente até a última semana. A $27^{\circ} \mathrm{C}$, ocorreu o mesmo para dx, sendo que terminam na $7^{\mathrm{a}}$ semana.

Os sarcofagídeos de regiões tropicais, apresentam diferenças biológicas quando estão em seus respectivos ambientes naturais, onde os fatores abióticos modificam-se constantemente ao longo do dia, e as suas necessidades alimentares não são limitadas ou excluídas, ao contrário do que ocorre quando criados em laboratório. No caso de S. trivittatus, acredita-se que as condições do Cerrado, onde ela é endêmica, influam marcadamente na sua bionômia. 
Aspectos Bionômicos de S. trivittatus em Laboratório • RJB Salviano et al.

\section{TABELA I}

Expectativa de vida de machos e fêmeas de Squamatoides trivittatus a 16 e $27 \pm 1^{\circ} \mathrm{C} / 50-60 \%$ e $70-80 \%$ de umidade relativa do ar

\begin{tabular}{rrrrrrrrrrrrr}
\hline \multicolumn{10}{c}{ Macho } \\
\hline \multicolumn{1}{c}{ x } & \multicolumn{1}{c}{$\mathrm{Lx}$} & \multicolumn{1}{c}{$\mathrm{dx}$} & \multicolumn{2}{c}{ Ex } & \multicolumn{2}{c}{$\mathrm{Tx}$} & \multicolumn{2}{c}{$\mathrm{ex}$} & \multicolumn{1}{c}{$100 \mathrm{qx}$} \\
\hline & $16^{\circ} \mathrm{C}$ & $27^{\circ} \mathrm{C}$ & $16^{\circ} \mathrm{C}$ & $27^{\circ} \mathrm{C}$ & $16^{\circ} \mathrm{C}$ & $27^{\circ} \mathrm{C}$ & $16^{\circ} \mathrm{C}$ & $27^{\circ} \mathrm{C}$ & $16^{\circ} \mathrm{C}$ & $27^{\circ} \mathrm{C}$ & $16^{\circ} \mathrm{C}$ & $27^{\circ} \mathrm{C}$ \\
\hline 1 & 1000 & 1000 & 120 & 285 & 940,0 & 857,5 & 3321,0 & 2019,0 & 3,32 & 2,02 & 12,0 & 28,5 \\
2 & 880 & 715 & 93 & 228 & 833,5 & 601,0 & 2381,0 & 1161,5 & 2,71 & 1,63 & 10,6 & 31,9 \\
3 & 787 & 487 & 280 & 244 & 647,0 & 365,0 & 1547,5 & 560,5 & 1,97 & 1,15 & 35,6 & 50,0 \\
4 & 507 & 243 & 160 & 169 & 427,0 & 158,5 & 900,5 & 195,5 & 1,78 & 0,81 & 31,6 & 69,7 \\
5 & 347 & 74 & 120 & 74 & 287,0 & 37,0 & 473,5 & 37,0 & 1,37 & 0,50 & 34,6 & 100,0 \\
6 & 227 & - & 54 & - & 4,0 & - & 186,5 & - & 0,82 & - & 47,1 & - \\
7 & 173 & - & 96 & - & 125,0 & - & 182,5 & - & 1,06 & - & 55,6 & - \\
8 & 77 & - & 58 & - & 48,0 & - & 57,5 & - & 0,75 & - & 75,0 & - \\
9 & 19 & - & 19 & - & 9,5 & - & 9,5 & - & 0,50 & - & 100,0 & - \\
10 & - & - & - & - & - & - & - & - & - & - & - & - \\
11 & - & - & - & - & - & - & - & - & - & - & - & - \\
12 & - & - & - & - & - & - & - & - & - & - & - & - \\
\hline
\end{tabular}

\begin{tabular}{|c|c|c|c|c|c|c|c|c|c|c|c|c|}
\hline \multicolumn{13}{|c|}{ Fêmea } \\
\hline $\mathrm{x}$ & \multicolumn{2}{|c|}{$\mathrm{Lx}$} & \multicolumn{2}{|c|}{$d x$} & \multicolumn{2}{|c|}{ Ex } & \multicolumn{2}{|c|}{$\mathrm{Tx}$} & \multicolumn{2}{|c|}{ ex } & \multicolumn{2}{|c|}{$100 \mathrm{qx}$} \\
\hline & $16^{\circ} \mathrm{C}$ & $27^{\circ} \mathrm{C}$ & $16^{\circ} \mathrm{C}$ & $27^{\circ} \mathrm{C}$ & $16^{\circ} \mathrm{C}$ & $27^{\circ} \mathrm{C}$ & $16^{\circ} \mathrm{C}$ & $27^{\circ} \mathrm{C}$ & $16^{\circ} \mathrm{C}$ & $27^{\circ} \mathrm{C}$ & $16^{\circ} \mathrm{C}$ & $27^{\circ} \mathrm{C}$ \\
\hline 1 & 1000 & 1000 & 67 & 378 & 966,5 & 811,0 & 3695,0 & 1692,0 & 3,70 & 1,69 & 6,7 & 37,8 \\
\hline 2 & 933 & 622 & 227 & 178 & 819,5 & 533,0 & 2728,5 & 881,0 & 2,92 & 1,42 & 24,3 & 28,6 \\
\hline 3 & 706 & 444 & 173 & 355 & 619,5 & 266,5 & 1909,0 & 348,0 & 2,70 & 0,78 & 24,5 & 80,0 \\
\hline 4 & 533 & 89 & 200 & 59 & 433,0 & 59,5 & 1289,5 & 81,5 & 2,42 & 0,92 & 37,5 & 66,7 \\
\hline 5 & 333 & 30 & 120 & 23 & 273,0 & 18,5 & 856,5 & 22,0 & 2,57 & 0,73 & 36,0 & 75,0 \\
\hline 6 & 213 & 7 & 27 & 7 & 199,5 & 3,5 & 583,5 & 3,5 & 2,74 & 0,50 & 12,5 & 100,0 \\
\hline 7 & 186 & - & 53 & - & 159,5 & - & 384,0 & - & 2,07 & - & 28,6 & - \\
\hline 8 & 133 & - & 67 & - & 99,5 & - & 224,5 & - & 1,69 & - & 50,0 & - \\
\hline 9 & 66 & - & 13 & - & 59,5 & - & 125,0 & - & 1,89 & - & 20,0 & - \\
\hline 10 & 53 & - & 27 & - & 39,5 & - & 65,5 & - & 1,24 & - & 50,0 & - \\
\hline 11 & 26 & - & 13 & - & 19,5 & - & 26,0 & - & 1,00 & - & 50,0 & - \\
\hline 12 & 13 & - & 13 & - & 6,5 & - & 6,5 & - & 0,50 & - & 100,0 & - \\
\hline
\end{tabular}

$\mathrm{x}$ : intervalo de idade em unidades de tempo (semanas); Lx: número de sobreviventes no começo da semana; dx: número de indivíduos mortos durante o intervalo etário $\mathrm{x}$; Ex: número de insetos vivos entre um dia e outro; Tx: número de insetos de idade $\mathrm{x}$ além da idade $\mathrm{x}$; ex: esperança de vida para os indivíduos de idade x; 100qx: razão da mortalidade por intervalo de idade.

\section{TABELA II}

Longevidade média para machos e fêmeas de Squamatoides trivittatus sob condições de temperatura e umidade constante $\left(16\right.$ e $27 \pm 1^{\circ} \mathrm{C} / 50-60 \%$ e $70-80 \%$ umidade relativa do ar)

\begin{tabular}{cccc}
\hline \multirow{2}{*}{ Temperatura } & \multicolumn{3}{c}{ Longevidade média (semanas) } \\
\cline { 2 - 4 } & Macho & Fêmea & Macho e fêmea \\
\hline $16^{\circ} \mathrm{C}$ & $3,5 \pm 2,0 \mathrm{a}$ & $3,8 \pm 2,6 \mathrm{a}$ & $1,9 \pm 1,2$ \\
& $(0,4-8,7)$ & $(0,4-10,8)$ & \\
$27^{\circ} \mathrm{C}$ & $2,1 \pm 1,3 \mathrm{~b}$ & $1,7 \pm 1,1 \mathrm{~b}$ & $3,7 \pm 0,7$ \\
& $(0,3-4,8)$ & $(0,3-5,8)$ & \\
\hline
\end{tabular}

Médias seguidas pelas mesmas letras não diferem entre si pelo teste "t" ao nível de 5\%; ( ) longevidade mínima e máxima em semanas.

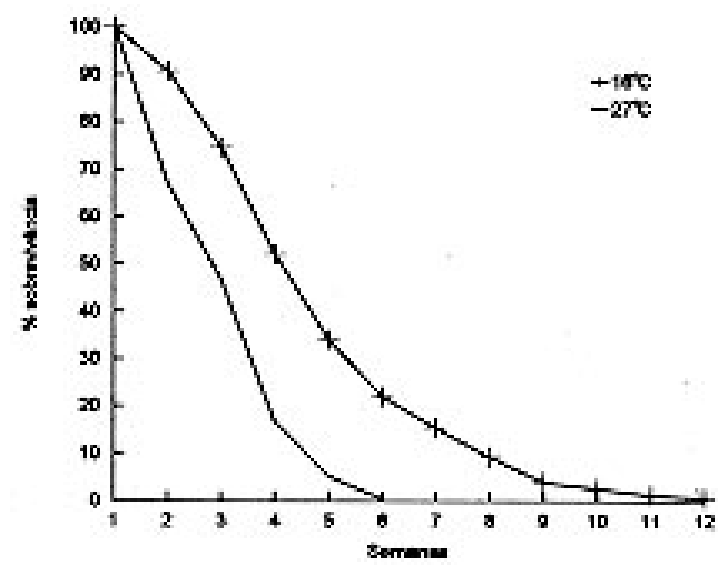

Fig. 2: curva de sobrevivência para Squamatoides trivittatus sob condições de temperatura e umidade constante (16 e $27 \pm 1^{\circ} \mathrm{C} / 50-60 \%$ e $70-80 \%$ de umidade relativa do ar). 


\section{AGRADECIMENTOS}

Ao Dr. Hugo de Souza Lopes, in memorian, pela iniciação nos estudo dos dípteros caliptrados, pelo apoio, incentivo e exemplo profissional. A Vanderléia Cristina de Oliveira, pela ajuda na manutenção das colônias. A Dra. Marli Maria Lima pela revisão e sugestões no texto.

\section{REFERÊNCIAS}

Baxter JA, Morrison PE 1983. Dynamics of growth modified by larval population in the flesh fly, Sarcophaga bullata. Can J Zool 61: 512-517.

Bressan S, Teles MC 1991. Longevidade e curva de sobrevivência de três espécies do gênero Anastrepha Schiner, 1868 (Diptera, Tephritidae) em laboratório. Rev bras Ent 35: 685-690.

Carey J 1987. Demographic methods in fruit fly research: survival, reproduction and mass-rearing. Ed. Global Fruit Working Group, California, 64pp.

d'Almeida JM 1983. Sinantropia de dípteros caliptrados na área metropolitana do Rio de Janeiro, Tese de Mestrado, UFRRJ, Rio de Janeiro, 193pp.

d'Almeida JM 1984. Sinantropia de Sarcophagidae (Diptera) na região metropolitana do Estado do Rio de Janeiro. Arq Univ Fed Rur Rio J 7: 101-110.

d'Almeida JM 1986. Substratos utilizados para a criação de dípteros caliptrados em uma área rural do Estado do Rio de Janeiro. Arq Univ Fed Rur Rio J 9: 13-22.

d'Almeida JM 1988. Substratos utilizados para a criação de dípteros caliptrados em uma área urbana do Município do Rio de Janeiro. Mem Inst Oswaldo Cruz 83: 201-206.

d'Almeida JM 1989. Substrato utilizados para a criação de dípteros caliptrados no Jardim Zoológico do Rio de Janeiro (RIO-ZOO). Mem Inst Oswaldo Cruz 84: 257-264.

d'Almeida JM 1993. Capture of calliptrate flies with different breeding substrates on beaches in Rio de Janeiro, RJ, Brazil. Mem Inst Oswaldo Cruz 88: 215220.

Ferraz MV 1992a. Estudos comparados do desenvolvimento biológico e do comportamento de Peckia chrysostoma (Wiedemann, 1830) $e$ Adiscochaeta ingens (Walker, 1849) (Diptera: Sarcophagidae) em condições de laboratório. Tese de Mestrado, Instituto Oswaldo Cruz, Rio de Janeiro, 138 pp.

Ferraz MV 1992b. Comparison of the reproductive between isolated Peckia chrysostoma (Wiedemann, 1930) and Adiscochaeta ingens (Walker, 1849) (Diptera: Sarcophagidae) females reared in laboratory. Mem Inst Oswaldo Cruz 87: 131-139.

Ferreira MJM 1978. Sinantropia de dípteros muscóides de Curitiba, Paraná, I. Calliphoridae. Rev Bras Biol 38: 445-454.

House HL 1977. Interaction between amino acids and glucose in larval nutrition of the fly Agria housei
(Diptera: Sarcophagidae) at low temperature. Can Ent 109: 87-91.

Jackson CHN 1936. Some new methods in the study of Glossina morsitans. Proc Zool Soc Lond 193: 811896.

Jirón LF, Bolaños R 1986. Biology and larval morphology by scannig electron microscopy of Panttonella intermutans Walker (Diptera, Sarcophagidae). Rev bras Ent 30: 27-30.

Kamal AS 1958. Comparative Study of Thirteen Species of Sarcosaprophagous Calliphoridae and Sarcophagidae (Diptera) I. Bionomic. Ann Ent Soc Am 51: 261-271.

Levot GW, Brown KR, Shipp E 1979. Larval growth of some calliphorid and sarcophagid Diptera. Bull Ent Res 69: 469-475.

Linhares AX 1979. Sinantropia de dípteros muscóides de Campinas. Tese de Mestrado. Universidade Estadual de Campinas, Campinas, SP, 129 p.

Linhares AX 1981. Synanthropy of Calliphoridae and Sarcophagidae (Diptera) in the city of Campinas, São Paulo, Brazil. Rev bras Ent 25: 189-215.

Lopes HS 1969. Family Sarcophagidae. In A Catalogue of the Diptera of the Americas South of the United States. Museu de Zoologia da Universidade de São Paulo. Fasc. 103, 88 pp.

Mackerras MJ 1933. Observations on the life-histories, nutrutional requirements and fecundity of blowflies. Bull Entomol Res 24: 353-562.

Madubunyl LC 1986. Laboratory life history parameters of the red-tailed fleshfly, Sarcophaga haemorrhoidalis (Fallen) (Diptera: Sarcophagidae). Insect Sci Appl 7: 617-621.

Mendes J 1991. Relação entre atratividade por iscas e estágios de desenvolvimento ovariano em fêmeas de dipteros muscóides sinantrópicos de Campinas, $S P$. Tese de Mestrado, Universidade Estadual de Campinas, Campinas, SP, 129 pp.

Mendes LOT 1961. Longevidade de Dysdercus. III. Machos Adultos de Dysdercus mendesi Bloete em condições de laboratório. Bragantia 20: 471-494.

Nishida K 1984. Experimental studies on estimation of postmortem interval by means of fly larvae infesting human cadavers. Nippon-Hoigaku-Zasshi 38: 2441.

Pearl R, Parker SL 1921. Experimental studies on the duration of life. I. Introductory discussion of the duration of life in Drosophila. Amer Nat 55: 481509.

Pearl R, Miner JR, Parker SL 1927. Experimental studies on the duration of life. XI. Density of population and life duration in Drosophila. Amer Nat 61: 289318.

Silveira Neto S, Nakano O, Barbin D, Nova NAV 1976. Manual de ecologia dos insetos. Agronômica Ceres, São Paulo, p. 243-245. 
254 Aspectos Bionômicos de S. trivittatus em Laboratório • RJB Salviano et al. 